\title{
Correction to: Diagnosis and Classification of Systemic Lupus Erythematosus
}

\author{
Sindhu R. Johnson and Martin Aringer
}

\section{Correction to:}

Chapter 3 in: Z. Touma (ed.), Outcome Measures and Metrics in Systemic Lupus Erythematosus, https://doi.org/10.1007/978-3-030-73303-2_3

In the original version of Chapter 3, page 59 had incorrect words Aplopezia and Negativ. These have now been corrected to "Alopecia and Negative". 